\title{
Research on Mobility management based on MIP table in mixed IPv4/v6 networks
}

\author{
Zheng Xiang \\ College of Medical Information Engineering, \\ Guangdong Pharmaceutical College \\ Guangzhou, 510006, China \\ rousseau2000@163.com
}

\author{
Zhengming Ma \\ College of Information Science and Technology \\ Sun Yat-sen University \\ Guangzhou, 510006, China \\ wartime2000@163.com
}

\begin{abstract}
IETF has specified Mobile IPv4 and Mobile IPv6 in RFC3344 and RFC3775 respectively, but not yet discussed Mobile IPv4/v6 in any published RFC. This paper proposes a scheme to solve one of Mobile IPv4/v6 problems which Home Agent (HA) locates in IPv6 network, and Correspondent Node (CN) locates in IPv4 network, while Mobile Node (MN) moves within IPv4 network. In the solution, a gateway called Mobile IPv4/v6 translation gateway (MIPv4/v6-TG) is introduced to bridge between IPv4 network and IPv6 network, which is made up of a traditional NAT-PT gateway and a Mobile IP application level gateway (MIP-ALG) built upon the NAT-PT gateway. MIP-ALG maintains a MIP table, a data structure, which is formed by entries. We use the MIP table to realize the communication between the IPv4 entities and the IPv6 entities. The creation, usage and update processes of MIP table are described in this paper. And it can work compatibly with RFC3344 and RFC3775.
\end{abstract}

Keywords-MobileIP(MIP;NAT-PT;Mobile IP application level gateway (MIP-ALG);Mobile IPv4/v6 translation gateway (MIPv4/v6-TG), MIP Table

\section{INTRODUCTION}

IETF specified the latest version of Mobile IPv4 [1] and Mobile IPv6[2] in RFC3344 and RFC3775 respectively. These two protocols work very well in pure IPv4 network and pure IPv6 network. However, both of them can not be directly applied to IPv4/v6 mixed networks. As the current network evolves gradually from IPv4 to IPv6, more and more mobile nodes need to roam in IPv4/v6 mixed networks. So we need to develop a transition scheme to support Mobile IP in IPv4/v6 mixed networks.

As a transition scheme, the solution should be developed based on the transition mechanisms for IP. Currently, three transition ways are recommended by IETF, namely, dual stacks (RFC4213) [3], tunneling (RFC3053) [4], and NATPT (RFC2766) [5][6]. There have been a lot of studies on Mobile IP in IPv4/v6 mixed networks, many of which are based on NAT-PT. An Internet Draft published by IETF [7] presents a solution to a situation of Mobile IPv4/v6, in which Home Agent (HA) and Mobile Node (MN) are in IPv6 network, Correspondent Node (CN) is in IPv4 network and NAT-PT gateway is located between IPv4 network and IPv6 network. It is proposed in this draft that NAT-PT do all of MIPv6 functionalilties on behalf of CN. This draft, however, deals with only part of the issues involved in Mobile IPv4/v6. In addition, this draft does not make clear how NAT-PT gateway does the functionalities on behalf of CN.

[8] studies the situation where both HA and CN are in IPv6 network and MN is in IPv4 network. A border router is placed between IPv4 network and IPv6 network to connect the two networks. After acquiring a new Care-of Address (CoA) in the IPv4 network, MN performs two registration processes, one is with the border router, and the other is with HAv6. Packets sent from CN to MN are encapsulated in new IPv6 packets which will be routed to the home network.

[9] studies the situation where HA is in IPv4 network, $\mathrm{CN}$ is in IPv6 network and MN moves within IPv4 network. A NAT-PT gateway is used to connect the two networks.

In [10], if HA is in IPv6 network, MN always registers its new CoA with $\mathrm{HA}$ by $\mathrm{BU}$ messages. If $\mathrm{MN}$ moves to IPv4 network, the BU messages sent by $\mathrm{MN}$ will be tunneled to NAT-PT gateway which decapsulates the messages and sends them to HA. Similarly, if HA is in IPv4 network, MN always registers its new CoA with HA by register request messages. If $\mathrm{MN}$ moves to IPv6 network, the register request messages sent by $\mathrm{MN}$ will be tunneled to NAT-PT gateway which decapsulates the messages and sends them to HA.

Mobile IPv4/v6 should be compatible with Mobile IPv4 and Mobile IPv6 because it is a transition scheme from Mobile IPv4 to Mobile IPv6. RFC3344 and RFC3775 are the schemes to MIPv4 and MIPv6 recommended by IETF. However, the solutions proposed in [8][9][10] do not pay enough attention to the compatibility with RFC3344 and RFC3775. This would prevent them from being chosen as a feasible transition scheme. This paper proposes a scheme to solve Mobile IPv4/v6 problems. The solution is based on Mobile IPv4/v6 Translation Gateway (MIPv4/v6-TG) which is made up of NAT-PT and a Mobile IP-Application Level Gateway (MIP-ALG). In the following sections, we will first describe the fundamental principles of our solution. Then we will introduce the data structure, MIP table, which is maintained by MIP-ALG, and describe in details how to create, use and update MIP table entries.

\section{TERMINOLOGY}

The terms frequently used in this paper are listed in TABLE1.In addition, "v4" and "v6" are used to indicate IPv4 and IPv6, respectively. If an IPv4 address is marked with a "\#”, it stands for an address from the address pool of NAT-PT gateway and is used to map an IPv6 address. If an 
IPv6 address is marked with a “*”, it means that this address is made up of a 96-bit NAT-PT prefix and an IPv4 address.

TABLE I. TERMS THAT ARE FREQUENTLY USED

\begin{tabular}{|c|c|c|c|}
\hline Terms & Meaning & Terms & Meaning \\
\hline MIP & Mobile IP & CNA & $\begin{array}{c}\text { Correspondent Node } \\
\text { Address }\end{array}$ \\
\hline HA & Home Agent & $B U$ & Binding Update \\
\hline$M N$ & Mobile Node & $B A$ & Binding Acknowledge \\
\hline$C N$ & Correspondent Node & HoTI & Home Test Init \\
\hline HAA & Home Agent Address & CoTI & Care-of Test Init \\
\hline HoA & Home Address & HoT & Home Test \\
\hline $\mathrm{CoA}$ & Care-of Address & CoT & Care-of Test \\
\hline
\end{tabular}

III. MIP TABLE

\section{A. $M I P v 4 / v 6-T G$}

MIPv4/v6-TG is made up of NAT-PT and MIP-ALG. On IPv6 network side, MIPv4/v6-TG acts as one of the Mobile IPv6 entities to combine with other MIPv6 entities inside the IPv6 network to form a complete MIPv6 model described in RFC3775. Inside the IPv6 network, the registration process and communication process can be performed as specified in RFC3775. Similarly, on IPv4 network side, MIPv4/v6-TG acts as one of the Mobile IPv4 entities to combine with other MIPv4 entities inside the IPv4 network to form a complete MIPv4 model described in RFC3344. Inside the IPv4 network, the registration process and communication process can be performed as specified in RFC3344.

As mentioned above, MIPv4/v6-TG will act as different MIP entities according to different combinations of the IP versions of $\mathrm{HA}, \mathrm{MN}$ and $\mathrm{CN}$. However, it can not decide which role it should act, if it does not know the IP versions of the three entities when MIPv4/v6-TG intercepts a MIPrelated message or datagram. Therefore, MIPv4/v6-TG should keep the information of the IP versions of the three entities and the bindings that are used when it acts as a particular MIP entity. In our solution, we introduce a new data structure called MIP table to solve this problem. We use 3-bit binary numbers to indicate the location where MN, HA and $\mathrm{CN}$ sit. If the communication entity location in the IPv6 network we record it as 1 , otherwise we record it as 0 .

The three MIP entities have eight kinds of IP version combinations, from 000 to 111 . At any time, there may be many MIP sessions, which correspond to the same scenario and thus use the same kind of MIP table entry. MIPv4/v6TG should be able to distinguish them so that the messages and datagrams can be sent to the right place. Therefore, each MIP table entry has four kinds of entrances: MIPv6 Message Entrance, MIPv6 Datagram Entrance, MIPv4 Message Entrance and MIPv4 Datagram Entrance. We will introduce the MIP table in the following.

\section{B. MIP table}

An MIP table entry should, typically, have the following fields.

Type: A three-bit field indicating the IP versions of HA, $\mathrm{MN}$ and $\mathrm{CN}$ respectively. For each bit, a value of 1 indicates the MIP entity is located in IPv6 network, while a value of 0 indicates the MIP entity is located in IPv4 network. Type value varies from 000 to 111 .

MIPv6 Message Entrance: A 128-bit field through which a particular MIP table entry can be found and accessed when a MIPv6 message is intercepted by MIPv4/v6-TG. Usually, this field is set to the IPv6 home address. All MIPv6 messages contain IPv6 home addresses. When a MIPv6 message is intercepted by MIPv4/v6-TG, MIPv4/v6-TG takes out the IPv6 home address from the intercepted message and uses it as an index to search the MIP table. In this process, MIPv4/v6-TG compares the IPv6 message entrance field of each entry with the IPv6 home address. If an entry is found, MIPv4/v6-TG will use the information recorded in the entry to process the intercepted message. If no entry is found, MIPv4/v6-TG will create a new entry.

MIPv6 Datagram Entrance: A 128-bit field through which a particular entry can be found and accessed when a MIPv6 datagram is intercepted by MIPv4/v6-TG. If an intercepted packet is not a MIPv6 message, MIPv4/v6-TG will take out its destination address and use the address as an index to search the MIP table. In this process, MIPv4/v6-TG compares the IPv6 datagram entrance filed of each entry with the address. If an entry is found, the intercepted packet is a MIPv6 datagram, and will be processed based on the information recorded in the entry. If no entry is found, the intercepted packet is not a MIPv6 datagram.

MIPv4 Message Entrance: A 32-bit field through which a particular entry can be found and accessed when a MIPv4 message is intercepted by MIPv4/v6-TG. On intercepting a MIPv4 message, MIPv4/v6-TG takes out its destination address and uses the address as an index to search for the corresponding MIP table entry. It is based on the MIP table entry that MIPv4/v6-TG processes the intercepted MIPv4 message.

MIPv4 Datagram Entrance: A 32-bit field through which a particular entry can be found and accessed when a MIPv4 datagram is intercepted by MIPv4/v6-TG. If an intercepted packet is not a MIPv4 message, MIPv4/v6-TG will take out its destination address and use the address as an index to search the MIP table. In this process, MIPv4/v6-TG compares the IPv4 datagram entrance filed of each entry with the address. If an entry is found, the intercepted packet is a MIPv4 datagram, and will be processed based on the information recorded in the entry. If no entry is found, the intercepted packet is not a MIPv4 datagram.

Cached Bindings: Bindings of home address and care-of address that are used by MIPv4/v6-TG when it acts as a particular MIP entity. Bindings may be of IPv4 form or of IPv6 form. Entries may have no binding, one binding, or two bindings. This depends on the types of the entries.

Source Port: A 16-bit field that records the source port value of Registration Request message, extended 
Registration Request message, or Agent Request message. All of these three messages are sent through UDP ports. When MIPv4/v6-TG intercepts such kind of message, it fills this field with the source port value. This field is used as the destination port when MIPv4/v6-TG sends back a reply later.

Destination Port: A 16-bit field that records the destination port value of Registration Request message, extended Registration Request message, or Agent Request message. It will be uses as the source port when MIPv4/v6TG sends back a reply later.

State: A 1-bit field indicating whether the entry is completed or not. There are two possible states for each entry: 1 (finished) and 0 (unfinished). An unfinished state indicates that the entry is now being created or updated. Entries with an unfinished state can not be used by MIPv4/v6-TG as a guide to process MIP datagrams.

Lifetime: A 16-bit field indicating entry lifetime. The meaning of this field depends on the value of the State field. When the State is 0 (unfinished), Lifetime means the entry should be completed before the time expires. When the State is 1 (finished), Lifetime means the number of seconds left before the entry is considered expired. In either case, when this filed becomes 0, MIPv4/v6-TG will delete the entry.

\section{APPLICATION EXAMPLE}

We will solve one of Mobile IPv4/v6 problems which Home Agent (HA) locates in IPv6 network, and Correspondent Node (CN) locates in IPv4 network, while Mobile Node (MN) moves within IPv4 network. And we will describe the creation, update and usage of type 100 MIP table entries as an example in the following.

\section{A. The create of Type 100 MIP Table Entries}

The creation of type 100 MIP table entry is triggered by Extended Registration Request message. The Extended Registration Request message is a new message introduced in this protocol. Its format is available in Fig.1.

\begin{tabular}{|c|c|c|c|c|c|c|c|c|c|}
\hline Type & S & B & D & M & G & r & T & x & Lifetime \\
\hline \multicolumn{4}{|c|}{ Home Address: 0} \\
\hline Home Agent Address: HAAv4\# \\
Care-of Address: CoAv4 \\
Identification \\
\hline Extension: CNAv4\# \\
Extension: HoAv6 \\
Other Extensions
\end{tabular}

Figure1. The extended Registration Request message.

In this protocol, MN sends the Extended Registration Request message only when it moves from IPv6 network to IPv4 network and performs the first registration. After MN's movement from IPv6 network to IPv4 network, the original entry becomes invalid. Therefore, a new entry should be created.
(1) On receiving the Extended Registration Request message, MIP-ALG can learn the IP versions of the three entities: HA is in IPv6 network, MN is in IPv4 network. As for $\mathrm{CN}$, if the IPv4 address of $\mathrm{CN}$ carried in the extension field of the message belongs to the NAT-PT address pool, the IP version of CN is IPv6. Otherwise, the IP version of $\mathrm{CN}$ is IPv4.

(2) MIP-ALG creates a new entry and sets the fields of Source Port and Destination Port to the source port number and destination port number of the intercepted message, respectively, the field of State to 0 , and the field of Lifetime to a value in which the creation of the entry must be accomplished.

(3) MIP-ALG sets the field of Type to 100, MIPv6 Message Entrance to HoAv6, MIPv4 Message Entrance to HAAv4\#, MIPv4 Datagram Entrance to HoAv4\#, Cached Bindings to HoAv4\# $\leftrightarrow$ CoAv4.

(4) MIP-ALG generates a BU message with CoAv6* and HAAv6 as its source address and destination address, respectively. HoAv6 is carried in the message. Here, HAAv6 is acquired by checking the NAT table using HAAv4\# as the index, while CoAv6* is acquired by adding a 96-bit NAT-PT prefix to CoAv4. Both HAAv4\# and CoAv4 are taken from the Extended Registration Request message. This message will be routed to HAv6.

(5) MIPv4/v6-TG intercepts the BA message replied from HAv6, takes out HoAv6 from the intercepted message as an index to search the MIP table and finds this entry.

(6) MIP-ALG generate Registration Reply message, with HAAv4\# and CoAv4 as its source address and destination address, respectively. This message is sent through UDP, with the source port number and destination port number copied from the Destination Port field and Source Port field. This message will be routed to MNv4.

(7) MIP-ALG sets the State field to 1 , and sets the Lifetime field to the lifetime of the binding.

\section{B. The update of Type 100 MIP Table Entries}

Note that when MN moves to a network of a different IP version, the original entry (if any) becomes invalid and a new MIP table entry should be created.

A Type 100 entry corresponds to a scenario where HA is located in IPv6 network, while MN and CN are located in IPv4 network. The update process of a Type 100 MIP table entry is as follows.

(1) MIPv4/v6-TG intercepts a Registration Request message, takes out destination address from the intercepted message as an index to search the MIP table and finds a related Type 4 entry.

(2) MIP-ALG sets the fields of State to 0, Lifetime to a value in which the update of this entry must be accomplished, and Source Port and Destination Port to the source port number and destination port number of the intercepted message.

(3)MIP-ALG updates Cached Bindings HoAv4\# $\leftrightarrow$ CoAv4 with the new CoAv4 carried in the Registration Request message.

(4) MIP-ALG generates a BU message based on the Registration Request message. The source address and 
destination address of the BU message are CoAv6* and HAAv6, respectively. HoAv6 is carried in the message. CoAv6* is acquired by adding a 96-bit NAT-PT prefix to CoAv4. HAAv6 and HoAv6 are acquired by checking the NAT table, using HAAv4\# and HoAv4\# as the index. This BU message will be routed to HAv6.

(5) MIPv4/v6-TG intercepts a BA message replied from HAv6, takes out HoAv6 from the intercepted message as an index to search the MIP table and finds this entry.

(6) MIP-ALG generates a Registration Reply message, with HAAv4\# and CoAv4 as the source address and destination address. This message is sent through UDP. The source port number and destination port number are copied from the Destination Port and Source Port, respectively. This message will be routed to MNv4.

(7) MIP-ALG sets the field of State to 1 , and the Lifetime to the lifetime of the binding.

\section{The usage of Type 100 MIP Table Entries}

The introduction of MIP table aims to maintain MIP sessions in IPv4/v6 mixed networks. When a datagram sent by $\mathrm{MN}$ or CN passes through MIPv4/v6-TG, MIPv4/v6-TG will take out the destination address of the datagram and uses it as an index to search the MIP table. If a matching entry is found, MIPv4/v6-TG will process the datagram, based on the information recorded in the entry.

When an IPv4 datagram passes through MIPv4/v6-TG, MIPv4/v6-TG will take out its destination address and uses it as an index to search the MIP table. If a Type 4 entry is found, MIPv4/v6-TG will process the datagram as follows.

(1)MIP-ALG encapsulates the datagram in a new IPv4 datagram. The source address of the outer IP header is copied from MIPv4 Message Entrance field. Then MIPALG uses the destination address of the inner IP header as an index to search Cached Bindings field, and takes out the care-of address bound with the destination address, and uses the care-of address as the destination address of the outer IP header.

(2)MIPv4/v6-TG sends the processed IPv4 datagram.

In the scenarios related to Type 100 entries, no MIPv6 datagrams will arrive at MIPv4/v6-TG.

\section{CONCLUSION}

The key to our solution is a MIP table, a newly introduced data structure. With the help of this gateway and the MIP table, RFC3344 and RFC3775 can be reused in IPv4 network and IPv6 network respectively. In this way, the Mobile IP entities in IPv4 network and the Mobile IP entities in IPv6 network can be transparent to each other.

Compared with other solutions, our solution has three main advantages. Firstly, it can work compatibly with RFC3344 and RFC3775. Secondly, our solution introduces MIP table. We can use the MIP table to realize the communication in IPv4/v6 mixed network easily. Thirdly, the creation, usage and update of the MIP table entries is easily too.
This work is supported in part by The Medium and Small Enterprise Technology Innovation Fund of The Ministry of Science and Technology (No. 09C26214402150), China.

\section{REFERENCES}

[1] Charles E. Perkins, Editor, “IP Mobility Support for IPv4”, RFC3344, IETF, August 2002

[2] Charles E. Perkins, Editor, "Mobility Support in IPv6”, RFC3775, IETF, June 2004

[3] E. Nordmark, "Basic Transition Mechanisms for IPv6 Hosts and Routers”, RFC4219, IETF, October 2005

[4] A. Durand, "IPv6 Tunnel Broker”, RFC3053, IETF, January 2001

[5] E. Nordmark, "Stateless IP/ICMP Translation Algorithm (SIIT)", RFC2765, IETF, February 2000

[6] G. Tsirtsis, P. Srisuresh, "Network Address Translation-Protocol Translation (NAT-PT)”, RFC2776, IETF, February 2000

[7] Joo- Chul Lee, “ Considerations for Mobility Support in NAT-PT”, Internet Draft, IETF, June 2005

[8] Changwen Liu, "Support mobile IPv6 in IPv4 domains", Vehicular Technology Conference, 2004. VTC 2004-Spring. 2004 IEEE 59th

[9] Dae Sun Kim; Choong Seon Hong; Suda, T, "A Terminal Mobility Management Architecture for IPv4 and IPv6 Environments”, Broadband Convergence Networks, 2006. BcN 2006. The 1st International Workshop on

[10] Hyun-Ho Choi; Dong-Ho Cho, "Mobility management based on mobile IP in mixed IPv4/IPv6 networks", Vehicular Technology Conference, 2003. VTC 2003-Fall. 2003 IEEE 58th

\section{ACKNOWLEDGMENT}

